\title{
Laser-excited fluorescence spectra of lanthanum monoxide
}

\author{
The $B^{2} \Sigma^{+} \rightarrow X^{2} \Sigma^{+}$system $^{\star}$
}

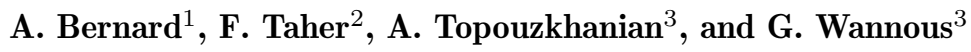 \\ 1 CRAL-Observatoire de Lyon, 9 avenue Charles André, F-69561 Saint-Genis-Laval Cedex, France \\ 2 Groupe de Physique Moléculaire et Applications, CNRS Libanais, Beyrouth, Liban \\ 3 Laboratoire de Spectrométrie Ionique et Moléculaire (LASIM), CNRS et Université Claude Bernard-Lyon I, 43 Bd. du Onze \\ Novembre 1918, F-69622 Villeurbanne Cedex, France
}

Received March 9; accepted May 26, 1999

\begin{abstract}
Fluorescence spectra of lanthanum monoxide excited by selected $\mathrm{Ar}^{+}$and $\mathrm{Kr}^{+}$laser lines have been analysed by Fourier transform spectrometry. Rotational levels in the $B^{2} \Sigma^{+}(v=2,3,4)$ electronic state have been populated either directly or after collisional relaxation, inducing many fluorescence transitions to the ground state $X^{2} \Sigma^{+}(v=1$ to 5$)$. Under our experimental conditions, bands of the $\Delta v=0$ sequence of the $B \rightarrow X$ system also appear, fairly developed.

A global treatment of the wavenumbers of some 830 lines in 9 bands of the system, involving the levels $v^{\prime}=0$ to 4 and $v^{\prime \prime}=0$ to 5 , is carried out. The consistent set of accurate effective spectroscopic constants for both electronic states, directly obtained at equilibrium, is expected to be useful for high resolution syntheses of S-type stellar spectra.
\end{abstract}

Key words: molecular data — line: identification

\section{Introduction}

The lanthanum monoxide molecule has long been recognized to play an important role in cool stellar atmosphere chemistry. Indeed, spectra of S-type red variables show $\mathrm{LaO}$ absorption bands of the red $(A-X)$ and yellow-green $(B-X)$ systems, particularly prominent at the light minimum. The strengths of the bands are very sensitive to the temperature (Keenan 1948; Bidelman 1953). In these stars, the abundance of La as an $s$-process element may be enhanced relatively to light elements, thus favoring the formation of LaO molecules (Wyckoff \& Clegg 1978; Lloyd

\footnotetext{
Send offprint requests to: A. Bernard

* Line lists are available in electronic form via http://wwwobs.univ-lyon1.fr/ ABernard/LaO
}

Evans \& Catchpole 1989). The C/O abundance ratio also appears as a determining parameter.

We have undertaken to reinvestigate some of the transitions of the $\mathrm{LaO}$ radical in the aim to obtain realistic molecular spectroscopic constants which are needed for the modeling of stellar opacities.

Seven electronic states of LaO, namely $X^{2} \Sigma^{+}, A^{\prime 2} \Delta$, $A^{2} \Pi, B^{2} \Sigma^{+}, C^{2} \Pi, D^{2} \Sigma^{+}$, and $F^{2} \Sigma^{+}$, are known from the observation of six band systems $(F-X, D-X, C-X$, $B-X, C-A^{\prime}$, and $\left.A-X\right)$, extending from ultraviolet to photographic infrared spectral regions (Huber \& Herzberg 1979; see also Carette 1990 and references therein). The $B-X$ system, which is the subject of the present work, was first analysed in rotation by Akerlind (1962), considering the ground state as a quartet $\left({ }^{4} \Sigma^{+}\right)$state. Only the $0-0,1-0$, and $0-1$ bands were concerned. This state was later definitely ascribed as ${ }^{2} \Sigma^{+}$(Green 1971). Schoonveld \& Sundaram (1974) estimated the vibrational intervals in both states, up to $v=11$, from the "band-head corrected" frequencies, and derived approximate values of the vibrational constants. Spin-rotation and hyperfine interactions were studied for $X^{2} \Sigma^{+}(v=0,1)$ and $B^{2} \Sigma^{+}(v=0)$ levels only (Bacis et al. 1973; Childs et al. 1986; Törring et al. 1988).

We report the observation and rotational analysis of a number of bands of the $B \rightarrow X$ system, involving higher vibrational levels in both states, up to $v^{\prime}=4$ and $v^{\prime \prime}=5$. Laser excitation technique has been used to obtain LaO fluorescence, and the spectra have been recorded at high resolution with a Fourier transform spectrometer. A consistent set of improved effective spectroscopic constants at equilibrium is determined. 
Table 1. Direct fluorescence lines observed upon laser excitation within the $B^{2} \Sigma^{+} \rightarrow X^{2} \Sigma^{+}$system of LaO

\begin{tabular}{ccc}
\hline Laser line & Excitation & Fluorescence \\
\hline \multirow{2}{*}{$\mathrm{Kr}^{+} 520.8-\mathrm{nm}$} & & \\
& $(2 \leftarrow 0) R_{23}(113)$ & $(2 \rightarrow 1,2) P_{23,24}(115), R_{23,24}(113)$ \\
& $(3 \leftarrow 1) R_{14}(49)$ & $(3 \rightarrow 2,3) P_{14,13}(51), R_{14,13}(49)$ \\
$\mathrm{Ar}^{+} 501.7-\mathrm{nm}$ & $(4 \leftarrow 1) R_{13}(10,23)$ & $(4 \rightarrow 3,4,5) P_{13,14}(12,25), R_{13,14}(10,23)$ \\
& $(4 \leftarrow 1) R_{14}(13,20)$ & $(4 \rightarrow 3,4,5) P_{14,13}(15,22), R_{14,13}(13,20)$ \\
& $(4 \leftarrow 1) R_{23}(44)$ & $(4 \rightarrow 3,4,5) P_{23,24}(46), R_{23,24}(44)$ \\
& $(4 \leftarrow 1) R_{24}(43)$ & $(4 \rightarrow 3,4,5) P_{24,23}(45), R_{24,23}(43)$ \\
\hline
\end{tabular}

\section{Experimental}

$\mathrm{LaO}$ vapor was obtained by heating a mixture of $\mathrm{LaI}_{3}$ powder with metallic lanthanum, in presence of traces of oxygen, in the tantalum heater of a special furnace filled with argon as a buffer gas. At the operating temperature, between 1600 and $1800 \mathrm{~K}$, the pressure was typically between 15 and 20 Torr. A description of the furnace can be found elsewhere (Shenyavskaya et al. 1973). LaO molecules were then excited by the light of a fixed-frequency argon-ion or krypton-ion laser which was beamed into the heater. Only $\mathrm{Ar}^{+}$501.7-nm and $\mathrm{Kr}^{+}$520.8-nm lines were found to give rise to resonance transitions in the spectral region of the $B \rightarrow X$ system. The induced fluorescence spectra were recorded from 16500 to $18600 \mathrm{~cm}^{-1}$, with a commercial Fourier transform spectrometer (Bomem DA $3)$. With this apparatus, a resolution of about $0.06 \mathrm{~cm}^{-1}$ is currently achieved, corresponding to an uncertainty of about $0.007 \mathrm{~cm}^{-1}$ in the determination of the positions of the sharpest lines. More details on the experimental procedure have been given by Shenyavskaya et al. (1993).

\section{Observations}

The $B^{2} \Sigma^{+} \rightarrow X^{2} \Sigma^{+}$system of LaO, lying in the greenyellow spectral range between 500 and $650 \mathrm{~nm}$, is composed of red-degraded, double-headed bands. Each head appears finely split (Fig. 1), this being a feature of the systems involving $X^{2} \Sigma^{+}$. It arises from the special $b_{\beta \mathrm{S}}$ coupling in the ground state, which splits each rotational level into two equidistant components separated by a constant value of about $0.5 \mathrm{~cm}^{-1}$ (see Sect. 4).

The fluorescence spectrum induced by the $\mathrm{Kr}^{+} 520.8$ $\mathrm{nm}$ and $\mathrm{Ar}^{+} 501.7-\mathrm{nm}$ laser lines consists of the pairs of strong lines $(P(J+1), R(J-1)$ patterns $)$ of the direct fluorescence, accompanied by many lines corresponding to transitions from upper state rotational levels populated by collisional transfer of energy. The $\mathrm{Kr}^{+}$520.8-nm line excites the $v=2$ and $v=3$ levels in $B^{2} \Sigma^{+}$from the ground state $v=0$ and $v=1$, respectively, giving rise to fluorescence transitions to $v=1,2$ and $v=2,3$ in $X^{2} \Sigma^{+}$. The $\mathrm{Ar}^{+}$501.7-nm line, which populates $v=4$ in $B^{2} \Sigma^{+}$from $X^{2} \Sigma^{+}(v=1)$, induces fluorescence transitions towards $X^{2} \Sigma^{+}(v=3,4,5)$. The relaxed spectrum is generally well developed, except for the $2 \leftarrow 0$ excitation by $\mathrm{Kr}^{+}$. The summary of excitation and direct fluorescence transitions is given in Table 1 . Parts of the observed fluorescence spectra are shown in Fig. 1.

Let us mention that under our experimental conditions the very first bands of the $\Delta v=0$ sequence are also observed, with rapidly decreasing intensity and development. The strong $0-0$, moderately intense $1-1$, and weak $2-2$ bands have been rotationally analysed.

\section{The $B^{2} \Sigma^{+} \rightarrow X^{2} \Sigma^{+}$transition}

The ground state of $\mathrm{LaO}$ has been proved to be of ${ }^{2} \Sigma^{+}$ symmetry, relevant of the $b_{\beta \mathrm{S}}$ coupling case. In this situation, the Fermi contact operator, $b \mathbf{I}$.S, couples the electron spin $\mathbf{S}$ to the nuclear spin $\mathbf{I}$. The total spin momentum $\mathbf{G}=\mathbf{I}+\mathbf{S}$ can take the values 3 and 4 , since $I=\frac{7}{2}$ for ${ }^{139} \mathrm{La}$ and $I=0$ for ${ }^{16} \mathrm{O}$, and $S=\frac{1}{2}$ for a doublet state. All rotational levels are then split into two components corresponding to $G=3$ and 4 , separated by four times the Fermi contact term $b$. For a given vibrational level $v$, the term values of the two sublevels have the form

$T_{3 v}(N)=T_{v}-\frac{9}{4} b+B_{v} N(N+1)-D_{v} N^{2}(N+1)^{2}$

and

$T_{4 v}(N)=T_{v}+\frac{7}{4} b+B_{v} N(N+1)-D_{v} N^{2}(N+1)^{2}$.

The upper state $B^{2} \Sigma^{+}$is in the more classical $b_{\beta \mathrm{J}}$ coupling $(\mathbf{J}=\mathbf{N}+\mathbf{S})$. The $F_{1}$ and $F_{2}$ rotational sublevels correspond to $N=J-\frac{1}{2}$ and $N=J+\frac{1}{2}$, respectively. The term values can be written as

$T_{1 v}(N)=T_{v}+B_{v} N(N+1)-D_{v} N^{2}(N+1)^{2}+\frac{\gamma_{v}}{2} N$

and

$T_{2 v}(N)=T_{v}+B_{v} N(N+1)-D_{v} N^{2}(N+1)^{2}-\frac{\gamma_{v}}{2}(N+1) .(4)$

The spin-rotation parameter $\gamma_{v}$ may be replaced, if necessary, by a polynomial expansion in $N(N+1)$, such as $\gamma_{v}+\gamma_{N} N(N+1)+\ldots$

A large hyperfine structure appears in each rotational level of $X^{2} \Sigma^{+}$, arising from the coupling $\mathbf{F}=\mathbf{N}+\mathbf{G}$ which gives $2 G+1$ hyperfine components. Likewise, for $B^{2} \Sigma^{+}$, each rotational level $J$ is composed of $2 I+1$ components $(\mathbf{F}=\mathbf{J}+\mathbf{I})$. Each band of such a ${ }^{2} \Sigma^{+} \rightarrow^{2} \Sigma^{+}$ 


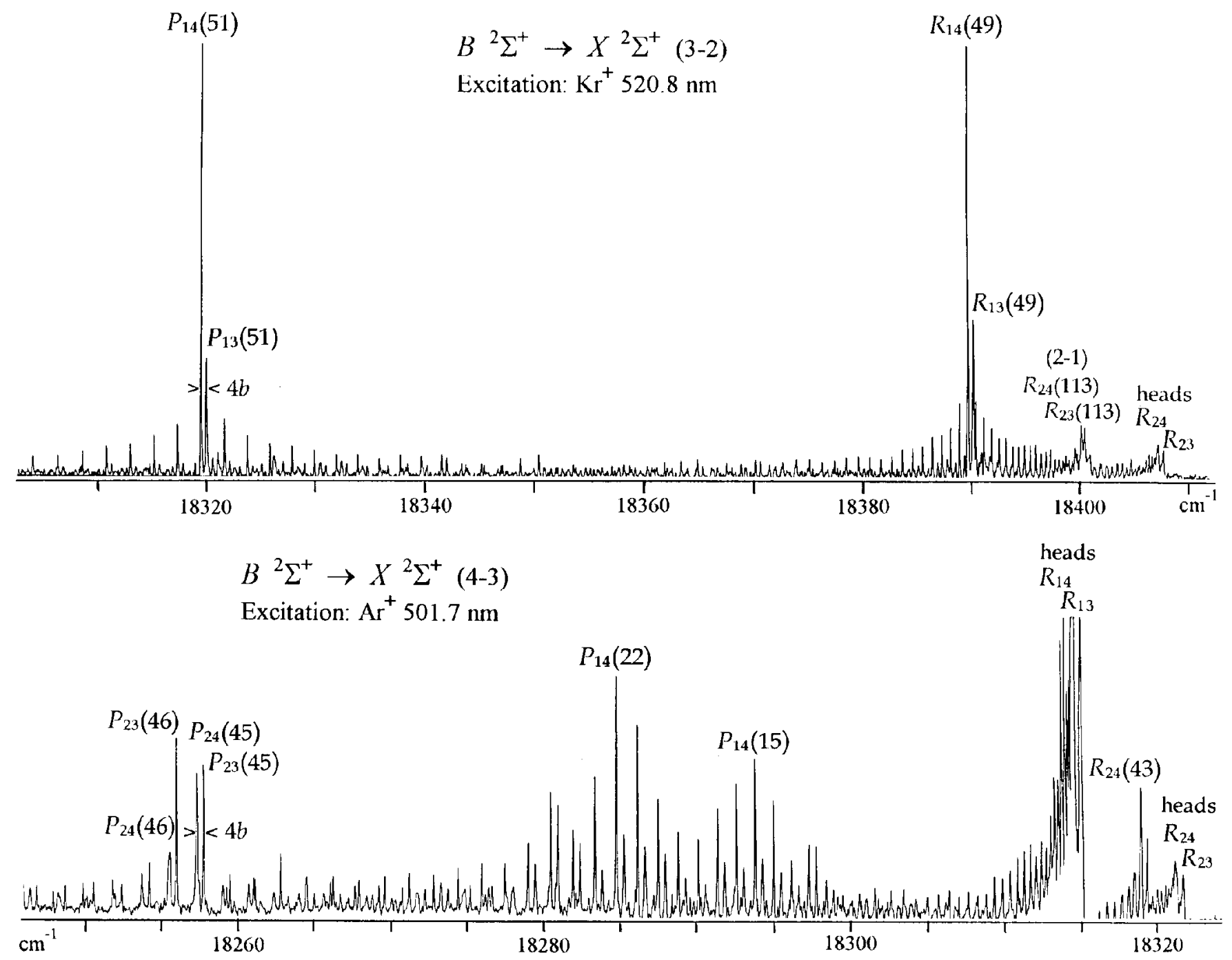

Fig. 1. The $B^{2} \Sigma^{+} \rightarrow X^{2} \Sigma^{+}(3-2)$ and $(4-3)$ relaxed bands following excitation by $\mathrm{Kr}^{+} 520.8-\mathrm{nm}$ and $\mathrm{Ar}^{+} 501.7-\mathrm{nm}$ laser lines. Some direct fluorescence lines are shown. $R_{23}(113)$ and $R_{24}(113)$ lines in the $(2-1)$ band are also noted

transition consists of 8 branches, following the selection rule $\Delta N= \pm 1$. The lines, which correspond to the unresolved transitions $(\Delta F=\Delta N)$ between the $F$ hyperfine sublevels, may show narrow or broadened profiles, according to the branch concerned. Thus, line profiles in the $R_{23}, R_{14}, P_{23}$, and $P_{14}$ branches are narrow, whereas they are asymmetrically broadened in $R_{24}, R_{13}, P_{24}$, and $P_{13}$, their widths increasing with rotation. Typical values of the FWHM's at mid-rotation $(J \approx 50)$ are about 0.040 and $0.130 \mathrm{~cm}^{-1}$, respectively (Bacis et al. 1973).

\section{Data processing and results}

The rotational analyses of the different bands raised no special difficulties since rotational constants for $X^{2} \Sigma^{+}$and $B^{2} \Sigma^{+}$states were available, at least for $v=0$ and $v=1$.
Thus, some 830 lines have been ascribed to the $0-0,1-1$, $2-1,2-2,3-2,3-3,4-3,4-4$, and $4-5$ bands.

In order to reduce the observational data to a unique and consistent set of spectroscopic constants a simultaneous multiband, non-linear least-squares adjustment has been performed. Approximate values of the parameters are first entered in the model, producing series of level energies in the upper and lower states from which line positions are calculated. Then, comparison between observations and model predictions allows corrections to these values to be determined. The process is iteratively continued until the standard deviations of successive fits differ by less than a small, preset value. Three or four iterations are generally sufficient for convergence to be achieved.

The rotational energies for the levels $v=0$ to 5 of $X^{2} \Sigma^{+}$and $v=0$ to 4 of $B^{2} \Sigma^{+}$are given by the formulae (1) to (4), where each parameter is expressed at 
Table 2. Equilibrium spectroscopic constants ${ }^{(a)}$ for the $X^{2} \Sigma^{+}$ and $B^{2} \Sigma^{+}$states of $\mathrm{LaO}$

\begin{tabular}{lll}
\hline Parameter & $X^{2} \Sigma^{+}$state & $B^{2} \Sigma^{+}$state \\
\hline$T_{\mathrm{e}}$ & 0 & $17878.733_{8}\left(2_{6}\right)^{(b)}$ \\
$\omega_{\mathrm{e}}$ & $817.026_{4}\left(7_{8}\right)$ & $734.281_{1}\left(8_{7}\right)$ \\
$\omega_{\mathrm{e}} x_{\mathrm{e}}$ & $2.129_{2}\left(2_{4}\right)$ & $1.992_{2}\left(2_{9}\right)$ \\
$10^{2} \omega_{\mathrm{e}} y_{\mathrm{e}}$ & $-0.31_{5}\left(2_{4}\right)$ & $-0.35_{4}\left(3_{2}\right)$ \\
$B_{\mathrm{e}}$ & $0.352520051^{(c)}$ & $0.3413316_{9}\left(9_{5}\right)$ \\
$10^{2} \alpha_{\mathrm{e}}$ & $0.14236516^{(c)}$ & $0.15422_{7}\left(3_{4}\right)$ \\
$10^{5} \beta_{\mathrm{e}}$ & $-0.2972390^{(c)}$ & \\
$10^{6} D_{\mathrm{e}}$ & $0.26258166^{(c)}$ & $0.2944_{7}\left(1_{2}\right)$ \\
$10^{9} \alpha_{\mathrm{D}}$ & $-0.226824^{(c)}$ & $-0.95_{9}\left(5_{0}\right)$ \\
$\gamma_{\mathrm{e}}$ & & $-0.25460_{6}\left(4_{7}\right)$ \\
$10^{3} \alpha_{\gamma}$ & & $-0.130_{1}\left(9_{4}\right)$ \\
$10^{6} \gamma_{\mathrm{N}}$ & & $-0.263_{2}\left(7_{6}\right)$ \\
$4 b$ & $0.465_{1}\left(1_{1}\right)$ & \\
$r_{\mathrm{e}}(\AA)$ & 1.8259 & 1.8556 \\
\hline
\end{tabular}

Note: $T_{\mathrm{v}}=T_{\mathrm{e}}+\omega_{\mathrm{e}}\left(v+\frac{1}{2}\right)-\omega_{\mathrm{e}} x_{\mathrm{e}}\left(v+\frac{1}{2}\right)^{2}+\omega_{\mathrm{e}} y_{\mathrm{e}}\left(v+\frac{1}{2}\right)^{3}$.

$B_{\mathrm{v}}=B_{\mathrm{e}}-\alpha_{\mathrm{e}}\left(v+\frac{1}{2}\right)+\beta_{\mathrm{e}}\left(v+\frac{1}{2}\right)^{2}$.

$D_{\mathrm{v}}=D_{\mathrm{e}}+\alpha_{\mathrm{D}}\left(v+\frac{1}{2}\right)$.

$\gamma_{v}=\gamma_{\mathrm{e}}-\alpha_{\gamma}\left(v+\frac{1}{2}\right)$.

(a) Constants are in $\mathrm{cm}^{-1}$, except $r_{\mathrm{e}}$ in Angström.

(b) $1 \sigma$ error in parentheses.

(c) Value from Törring et al. (1988), fixed in the present fit.

equilibrium as a polynomial expansion in $v+\frac{1}{2}$. The signs of the coefficients are defined as usual (Huber \& Herzberg 1979). Thus, the wavenumbers of all 830 lines belonging to the nine above-mentioned bands could be directly reduced to the set of effective equilibrium constants given in Table 2.

It has been verified that the values found for the rotational constants in the ground state are consistent, within \pm one standard deviation limits or better, with the values from Törring et al. (1988). In the final fit, the parameters were kept fixed at these values, which were determined very accurately from the study of the microwave absorption spectrum. The $b$ parameter in $X^{2} \Sigma^{+}$ and spin-rotation parameters in $B^{2} \Sigma^{+}$were left free to vary, since they appear as effective in the present model which does not include hyperfine terms (except $b \mathbf{I} . \mathbf{S}$ ).
Thus, small deviations are observed between the present values and the true values reported previously for these parameters (Bacis et al. 1973; Childs et al. 1986; Törring et al. 1988). The dependence with $v$ and $N$ of the effective spin-rotation constant in the upper state is found to be significant.

Values of the vibrational constants in $X^{2} \Sigma^{+}$and $B^{2} \Sigma^{+}$ states, and of the rotational constants in $B^{2} \Sigma^{+}$are obtained, one or two orders of magnitude more precise than earlier values. This set of spectroscopic constants allows the positions of the observed lines to be calculated to nearly the experimental precision (the standard deviation of the fit is $0.009 \mathrm{~cm}^{-1}$ ). It is expected to yield a realistic representation of the band spectrum up to $N$ 's of about 90 in the range of the $v$ 's considered, and thus to be useful for high resolution syntheses of S-type stellar spectra. Line wavenumbers corresponding to the 14 bands with FranckCondon intensity factors greater than 0.10 have been calculated. These data are available in electronic form (see footnote to the title).

Work is in progress at the LASIM concerning the red $A^{2} \Pi \rightarrow X^{2} \Sigma^{+}$and infrared $A^{2} \Pi \rightarrow A^{\prime 2} \Delta$ transitions.

\section{References}

Åkerlind L., 1962, Ark. Fys. 22, 55

Bacis R., Collomb R., Bessis N., 1973, Phys. Rev. A 8, 2255

Bidelman W.P., 1953, ApJ 117, 377

Carette P., 1990, J. Mol. Spectrosc. 140, 269

Childs W.J., Goodman G.L., Goodman L.S., Young L., J. Mol. Spectrosc. 119, 166

Green D.W., 1971, J. Mol. Spectrosc. 38, 155

Huber K.P., Herzberg G., 1979, Molecular Spectra and Molecular Structure IV, Constants of Diatomic Molecules. Van Nostrand-Reinhold, New-York

Keenan P.C., 1948, ApJ 107, 420

Lloyd Evans T., Catchpole R.M., 1989, MNRAS 237, 219

Schoonveld L., Sundaram S., 1974, ApJS 27, 307

Shenyavskaya E.A., Egorova I.V., Lupanov V.N., 1973, J. Mol. Spectrosc. 47, 355

Shenyavskaya E.A., Ross A.J., Topouzkhanian A., Wannous G., 1993, J. Mol. Spectrosc. 162, 327

Törring T., Zimmermann K., Hoeft J., 1988, Chem. Phys. Lett. 151,520

Wyckoff S., Clegg R.E.S., 1978, MNRAS 184, 127 